MATHEMATICS OF COMPUTATION

Volume 68, Number 225, January 1999, Pages 169-186

S 0025-5718(99)00999-0

\title{
CONVERGENCE OF NEWTON'S METHOD AND INVERSE FUNCTION THEOREM IN BANACH SPACE
}

\author{
WANG XINGHUA
}

\begin{abstract}
Under the hypothesis that the derivative satisfies some kind of weak Lipschitz condition, a proper condition which makes Newton's method converge, and an exact estimate for the radius of the ball of the inverse function theorem are given in a Banach space. Also, the relevant results on premises of Kantorovich and Smale types are improved in this paper.
\end{abstract}

We continue to discuss the problem of convergence in the Newton method

$$
x_{n+1}=x_{n}-f^{\prime}\left(x_{n}\right)^{-1} f\left(x_{n}\right), \quad n=0,1, \cdots,
$$

to solve an operator equation $f$ which maps from some domain $D$ in a real or complex Banach space $\mathbf{X}$ to another Banach space $\mathbf{Y}$,

$$
f(x)=0 .
$$

Now we come back to the problem which we bypassed in [1].

We always assume that $f^{\prime}\left(x_{0}\right)^{-1}$ exists and $f^{\prime}\left(x_{0}\right)^{-1} f^{\prime}$ satisfies some kind of Lipschitz condition similar to that of [1] in some open ball $\mathrm{B}\left(x_{0}, r\right) \subset D$ with center $x_{0}$ and radius $r$ (or some closed ball $\overline{\mathrm{B}\left(x_{0}, r\right)} \subset D$ ) in order to study the convergence of Newton's method and the domain of the local inverse function of $f$ at $x_{0}$.

\section{The DOMAIN OF THE INVERSE FUnCTION}

The inverse function theorem asserts that there is an inverse function $f_{x_{0}}^{-1}$ defined on some open ball $\mathrm{B}\left(f\left(x_{0}\right), \varepsilon\right) \subset \mathbf{Y}$ with the property that

$$
\begin{gathered}
f_{x_{0}}^{-1}\left(f\left(x_{0}\right)\right)=x_{0}, \\
f\left(f_{x_{0}}^{-1}(y)\right)=y, \quad \forall y \in \mathrm{B}\left(f\left(x_{0}\right), \varepsilon\right),
\end{gathered}
$$

and $f_{x_{0}}^{-1}$ is differentiable. Now we study the exact lower bound estimate of the radius of this ball.

For this reason, we assume that $f$ has a continuous derivative in the ball $\mathrm{B}\left(x_{0}, r\right)$, $f^{\prime}\left(x_{0}\right)^{-1}$ exists and $f^{\prime}\left(x_{0}\right)^{-1} f^{\prime}$ satisfies the center Lipschitz condition with the $L$ average,

$$
\left\|f^{\prime}\left(x_{0}\right)^{-1} f^{\prime}(x)-\mathrm{I}\right\| \leq \int_{0}^{\rho(x)} L(u) d u, \quad \forall x \in \mathrm{B}\left(x_{0}, r\right)
$$

Received by the editor March 12, 1997 and, in revised form, June 6, 1997.

1991 Mathematics Subject Classification. Primary 65H10.

Supported by the China State Major Key Project for Basic Research and the Zhejiang Provincial Natural Science Foundation. 
where $\rho(x)=\left\|x-x_{0}\right\|$ and $L$ is a positive integrable function in the interval $(0, r)$. By Banach's theorem, when $r_{0} \leq r$, for all $x \in \mathrm{B}\left(x_{0}, r_{0}\right), f^{\prime}(x)^{-1}$ exists and

$$
\left\|f^{\prime}(x)^{-1} f^{\prime}\left(x_{0}\right)\right\| \leq \frac{1}{1-\int_{0}^{\rho(x)} L(u) d u},
$$

where $r_{0}$ satisfies

$$
\int_{0}^{r_{0}} L(u) d u=1
$$

Theorem 1.1. Suppose that $r \geq r_{0}$ and $b=\int_{0}^{r_{0}} L(u) u d u$. Then under the hypothesis of condition (1.1), we have

$$
\mathrm{B}\left(f\left(x_{0}\right), b /\left\|f^{\prime}\left(x_{0}\right)^{-1}\right\|\right) \subset f\left(\mathrm{~B}\left(x_{0}, r_{0}\right)\right),
$$

and in the open ball on the left, $f_{x_{0}}^{-1}$ exists and is differentiable. Moreover, the radius of the ball is the best possible.

Lemma 1.2. Let

$$
h(t)=\beta-t+\int_{0}^{t} L(u)(t-u) d u, \quad 0 \leq t \leq R,
$$

where $R$ satisfies

$$
\frac{1}{R} \int_{0}^{R} L(u)(R-u) d u=1 .
$$

Then when $0<\beta<b, h$ is decreasing monotonically in $\left[0, r_{0}\right]$, while it is increasing monotonically in $\left[r_{0}, R\right]$ and

$$
h(\beta)>0, \quad h\left(r_{0}\right)=\beta-b<0, \quad h(R)=\beta>0 .
$$

Moreover, $h$ has a unique zero in each interval, denoted by $r_{1}$ and $r_{2}$. They satisfy

$$
\beta<r_{1}<\frac{r_{0}}{b} \beta<r_{0}<r_{2}<R .
$$

Proof. It is obvious by the sign of $h^{\prime}(t)=-1+\int_{0}^{t} L(u) d u$ that $h(t)$ is piecewise monotone. By the positivity of $L$, we see that $\varphi(t):=\frac{1}{t} \int_{0}^{t} L(u)(t-u) d u$ is increasing monotonically with respect to $t$. In fact, for $0<t_{1}<t_{2}$,

$$
\begin{aligned}
\varphi\left(t_{2}\right)-\varphi\left(t_{1}\right) & =\int_{t_{1}}^{t_{2}} L(u) d u-\left(\frac{1}{t_{2}} \int_{t_{1}}^{t_{2}}+\left(\frac{1}{t_{2}}-\frac{1}{t_{1}}\right) \int_{0}^{t_{1}}\right) L(u) u d u \\
& \geq \int_{t_{1}}^{t_{2}} L(u) d u-\int_{t_{1}}^{t_{2}} L(u) d u-\left(\frac{1}{t_{2}}-\frac{1}{t_{1}}\right) \int_{0}^{t_{1}} L(u) u d u \\
& =\left(\frac{1}{t_{1}}-\frac{1}{t_{2}}\right) \int_{0}^{t_{1}} L(u) u d u>0 .
\end{aligned}
$$

Thus we have

$$
\begin{aligned}
\beta & <r_{1}=h\left(r_{1}\right)+r_{1}=\beta+\varphi\left(r_{1}\right) r_{1}<\beta+\varphi\left(r_{0}\right) r_{1} \\
& =\beta+\frac{1}{r_{0}} \int_{0}^{r_{0}} L(u)\left(r_{0}-u\right) d u \cdot r_{1}=\beta+r_{1}-\frac{b}{r_{0}} r_{1} .
\end{aligned}
$$


By this lemma, Theorem 1.1 implies a more precise proposition, as follows. For this purpose, we assume the inequality (1.1) can be extended to the boundary, i.e.

$$
\left\|f^{\prime}\left(x_{0}\right)^{-1} f^{\prime}(x)-\mathrm{I}\right\| \leq \int_{0}^{\rho(x)} L(u) d u, \quad \forall x \in \overline{\mathrm{B}\left(x_{0}, r\right)} .
$$

Proposition 1.3. Suppose that $r \geq r_{1}$ and $0<\beta<b=\int_{0}^{r_{0}} L(u) u d u$, where $r_{1}$ is determined by Lemma 1.2. Then, under the hypothesis of the condition $\left(1.1^{\prime}\right)$,

$$
\overline{\mathrm{B}\left(f\left(x_{0}\right), \beta /\left\|f^{\prime}\left(x_{0}\right)^{-1}\right\|\right)} \subset f\left(\overline{\mathrm{B}\left(x_{0}, r_{1}\right)}\right),
$$

and in the closed ball on the left, $f_{x_{0}}^{-1}$ exists, is differentiable, and its derivative $\left(f_{x_{0}}^{-1}\right)^{\prime}(y)=f^{\prime}(x)^{-1}$ at $y=f(x)$ satisfies $(1.2)$. Moreover, as a closed ball of the image, the radius $r_{1}$ is as small as possible.

Proof. Arbitrarily choosing

$$
y \in \overline{\mathrm{B}\left(f\left(x_{0}\right), \beta /\left\|f^{\prime}\left(x_{0}\right)^{-1}\right\|\right)},
$$

we consider two sequences $\left\{x_{n}\right\} \subset \mathbf{X}$ and $\left\{t_{n}\right\} \subset \mathbf{R}$, respectively given by

$$
x_{n+1}=x_{n}-f^{\prime}\left(x_{0}\right)^{-1}\left(f\left(x_{n}\right)-y\right), \quad n=0,1, \cdots,
$$

and

$$
t_{n+1}=t_{n}+h\left(t_{n}\right), \quad t_{0}=0, \quad n=0,1, \cdots .
$$

First, by the fact that $h(t)+t$ increases monotonically with respect to $t$ and $t_{0}=0<t_{1}=\beta<r_{1}$, we inductively find that $\left\{t_{n}\right\}$ increases monotonically and is less than $r_{1}$. Thus $\left\{t_{n}\right\}$ converges to $r_{1}$.

Then, by induction, for all $n$ we will prove that

$$
\left\|x_{n+1}-x_{n}\right\| \leq t_{n+1}-t_{n} .
$$

By (1.9) and (1.7),

$$
\left\|x_{1}-x_{0}\right\| \leq\left\|f^{\prime}\left(x_{0}\right)^{-1}\right\| \cdot\left\|f\left(x_{0}\right)-y\right\| \leq \beta=t_{1}-t_{0} .
$$

This means (1.12) is true for $n=0$. Suppose that (1.12) is valid until some $n-1$. For $0 \leq \tau \leq 1$, let

$$
\begin{aligned}
& x_{n-1+\tau}=x_{n-1}+\tau\left(x_{n}-x_{n-1}\right), \\
& t_{n-1+\tau}=t_{n-1}+\tau\left(t_{n}-t_{n-1}\right) .
\end{aligned}
$$

We have

$$
\begin{aligned}
\left\|x_{n-1+\tau}-x_{0}\right\| & \leq\left\|x_{1}-x_{0}\right\|+\cdots+\left\|x_{n-1}-x_{n-2}\right\|+\tau\left\|x_{n}-x_{n-1}\right\| \\
& \leq\left(t_{1}-t_{0}\right)+\cdots+\left(t_{n-1}-t_{n-2}\right)+\tau\left(t_{n}-t_{n-1}\right) \\
& =t_{n-1+\tau}<r_{1} \leq r
\end{aligned}
$$

Thus, by virtue of the equality

$$
\begin{aligned}
x_{n+1}-x_{n} & =-f^{\prime}\left(x_{0}\right)^{-1}\left(f\left(x_{n}\right)-f\left(x_{n-1}\right)-f^{\prime}\left(x_{0}\right)\left(x_{n}-x_{n-1}\right)\right) \\
& =-\int_{0}^{1}\left(f^{\prime}\left(x_{0}\right)^{-1} f^{\prime}\left(x_{n-1+\tau}\right)-\mathrm{I}\right)\left(x_{n}-x_{n-1}\right) d \tau
\end{aligned}
$$


and (1.1), we obtain

$$
\begin{aligned}
\left\|x_{n+1}-x_{n}\right\| & \leq \int_{0}^{1}\left\|f^{\prime}\left(x_{0}\right)^{-1} f^{\prime}\left(x_{n-1+\tau}\right)-\mathrm{I}\right\|\left\|x_{n}-x_{n-1}\right\| d \tau \\
& \leq \int_{0}^{1} \int_{0}^{\rho\left(x_{n-1+\tau}\right)} L(u) d u\left\|x_{n}-x_{n-1}\right\| d \tau \\
& \leq \int_{0}^{1} \int_{0}^{t_{n-1+\tau}} L(u) d u\left(t_{n}-t_{n-1}\right) d \tau \\
& =\int_{0}^{t_{n}} L(u)\left(t_{n}-u\right) d u-\int_{0}^{t_{n-1}} L(u)\left(t_{n-1}-u\right) d u \\
& =t_{n+1}-t_{n} .
\end{aligned}
$$

This indicates that (1.12) is valid for all $n$.

The inequality (1.12) above shows that the sequence $\left\{x_{n}\right\}$ is self-convergent and so is convergent. Taking the limit on both sides in (1.10), we see that $x=\lim x_{n}$ satisfies

$$
f(x)=y
$$

Also, since $\left\|x_{n}-x_{0}\right\| \leq r_{1}$, we have

$$
x=f_{x_{0}}^{-1}(y) \in \overline{\mathrm{B}\left(x_{0}, r_{1}\right)} .
$$

For this reason we have to prove $x$ satifying (1.14) is unique in the closed ball. This will be given togather with the proof of the next proposition. Finally, the differentiablity of the inverse function follows by (1.2).

Remark. Except for the differentiablity of the inverse function, the proposition is also true for $\beta=b$.

Besides Proposition 1.3, we have the following proprosition, which is called the branch separation theorem

Proposition 1.4. Suppose that $r_{1} \leq r<r_{2}$ and $0<\beta<b$, where $r_{1}, r_{2}$ and $b$ are determined by Lemma 1.2 and Theorem 1.1. Then, under the condition (1.1'),

$$
\overline{\mathrm{B}\left(f\left(x_{0}\right), \beta /\left\|f^{\prime}\left(x_{0}\right)^{-1}\right\|\right)} \bigcap f\left(\mathrm{~B}\left(x_{0}, r\right) \backslash \overline{\mathrm{B}\left(x_{0}, r_{1}\right)}\right)=\emptyset .
$$

Proof. Arbitrarily choose

$$
y \in \mathrm{B}\left(f\left(x_{0}\right), \beta /\left\|f^{\prime}\left(x_{0}\right)^{-1}\right\|\right), \quad x_{0}^{\prime} \in \mathrm{B}\left(x_{0}, r\right) .
$$

Let

$$
\begin{aligned}
& x_{n+1}^{\prime}=x_{n}^{\prime}-f^{\prime}\left(x_{0}\right)^{-1}\left(f\left(x_{n}^{\prime}\right)-y\right), \quad n=0,1, \cdots, \\
& t_{n+1}^{\prime}=t_{n}^{\prime}+h\left(t_{n}^{\prime}\right), \quad t_{0}^{\prime}=\left\|x_{0}^{\prime}-x_{0}\right\|, \quad n=0,1, \cdots .
\end{aligned}
$$

Since

$$
x_{n+1}^{\prime}-x_{n+1}=-\int_{0}^{1}\left(f^{\prime}\left(x_{0}\right)^{-1} f^{\prime}\left(x_{n}+\tau\left(x_{n}^{\prime}-x_{n}\right)\right)-\mathrm{I}\right)\left(x_{n}^{\prime}-x_{n}\right) d \tau,
$$

we can prove that

$$
\left\|x_{n}^{\prime}-x_{n}\right\| \leq t_{n}^{\prime}-t_{n}, \quad n=0,1, \cdots .
$$

Hence, $\left\{x_{n}^{\prime}\right\}$ is also convergent to $x=\lim x_{n}$. Therefore, there is only one $x \in$ $\overline{\mathrm{B}\left(x_{0}, r_{1}\right)}$ in the open ball $\mathrm{B}\left(x_{0}, r\right)$ that satisfies (1.14). 
Remark. The proof of Propositions 1.3 and 1.4 may be viewed as the proof of the existence and uniqueness theorems about the solution of the equation $f(x)=y$, and the premise (1.9) and (1.17) can be replaced by

$$
\left\|f^{\prime}\left(x_{0}\right)^{-1}\left(f\left(x_{0}\right)-y\right)\right\| \leq \beta \text {. }
$$

Hence, setting $y=0$ and $\beta=\left\|f^{\prime}\left(x_{0}\right)^{-1} f\left(x_{0}\right)\right\|$, we have

Theorem 1.5. Let $\beta=\left\|f^{\prime}\left(x_{0}\right)^{-1} f\left(x_{0}\right)\right\| \leq b$. Assume that $r_{1} \leq r<r_{2}$ if $\beta<b$, or $r=r_{1}$ if $\beta=b$, where $r_{1}, r_{2}$ and $b$ are determined by Lemma 1.2 and Theorem 1.1. Then, under the conditions $\left(1.1^{\prime}\right)$, the equation (0.2) has a unique solution

$$
x^{*} \in \overline{B\left(x_{0}-f^{\prime}\left(x_{0}\right)^{-1} f\left(x_{0}\right), r_{1}-\beta\right)} \subset \overline{B\left(x_{0}, r_{1}\right)}
$$

in the closed ball $\overline{\mathrm{B}\left(x_{0}, r\right)}$.

\section{FURTHER DISCUSSION OF LIPSCHITZ CONDITIONS}

In the ball $\mathrm{B}\left(x_{0}, r\right)$, the Lipschitz condition with the constant $L$ is

$$
\left\|f(x)-f\left(x^{\prime}\right)\right\| \leq L\left\|x-x^{\prime}\right\|,
$$

where $x, x^{\prime} \in \mathrm{B}\left(x_{0}, r\right)$. If (2.1) is only true for all $x \in \mathrm{B}\left(x_{0}, r\right)$ and $x^{\prime}=x_{0}$, then it is called the center Lipschitz condition in [1]; if (2.1) is valid for all $x^{\prime} \in \mathrm{B}\left(x_{0}, r\right)$ and for all $x=x_{0}+\tau\left(x^{\prime}-x_{0}\right)(0 \leq \tau \leq 1)$, then it is called the radius Lipschitz condition. Now, if (2.1) is valid for all $x \in \mathrm{B}\left(x_{0}, r\right)$ and for all $x^{\prime} \in \mathrm{B}(x, r-\rho(x))$, then we call it the center Lipschitz condition in the inscribed sphere. For a constant or positive integrable function $L$, among all Lipschitz conditions with the constant $L$ or the average of $L$, there is an implication relation $\succ$ as follows:

Lipschitz condition in a ball

$\succ$ the center Lipschitz condition in the inscribed sphere

$\succ$ the radius Lipschitz condition

$\succ$ the center Lipschitz condition.

Custom is the only reason why we give the names of different Lipschitz conditions. It is not necessary in essence; see Theorems 6.3 and 6.4 in [1]. Sometimes, however, we have to pay attention to such accustomed thinking because it determines the development of the literature.

\section{Convergence of Newton's method}

Suppose that $f$ has a continuous derivative in the closed ball $\overline{\mathrm{B}\left(x_{0}, r\right)}, f^{\prime}\left(x_{0}\right)^{-1}$ exists and $f^{\prime}\left(x_{0}\right)^{-1} f^{\prime}$ satisfies the center Lipschitz condition in the inscribed sphere with the average of $L$,

$$
\begin{gathered}
\left\|f^{\prime}\left(x_{0}\right)^{-1}\left(f^{\prime}(x)-f^{\prime}\left(x^{\prime}\right)\right)\right\| \leq \int_{\rho(x)}^{\rho\left(\overline{x x^{\prime}}\right)} L(u) d u, \\
\forall x \in \mathrm{B}\left(x_{0}, r\right), \quad \forall x^{\prime} \in \overline{\rho m B}(x, r-\rho(x)),
\end{gathered}
$$

where $\rho(x)=\left\|x-x_{0}\right\|, \rho\left(\overline{x x^{\prime}}\right)=\rho(x)+\left\|x^{\prime}-x\right\| \leq r$, and $L$ is a positive nondecreasing function in $[0, r]$. Under this hypothesis, the conditions (1.1) and $\left(1.1^{\prime}\right)$ are of course satisfied, and thus Theorems 1.1 and 1.5, Propositions 1.3 and 1.4 hold. 
Theorem 3.1. Assume that $\beta=\left\|f^{\prime}\left(x_{0}\right)^{-1} f\left(x_{0}\right)\right\| \leq b$ and $r \geq r_{1}$, where $b$ and $r_{1}$ are determined by Theorem 1.1 and Lemma 1.2. Then, under the condition (3.1), Newton's method (0.1) is defined for all $n$ and converges to a solution $x^{*}$ of equation $(0.2)$,

$$
x^{*} \in \overline{\mathrm{B}\left(x_{1}, r_{1}-\beta\right)} \subset \overline{\mathrm{B}\left(x_{0}, r_{1}\right)} .
$$

Moreover, for all $n \geq n_{0} \geq 0$, the best possible error bounds

$$
\left\|x^{*}-x_{n}\right\| \leq\left(r_{1}-t_{n}\right)\left(\frac{\left\|x^{*}-x_{n_{0}}\right\|}{r_{1}-t_{n_{0}}}\right)^{2^{n-n_{0}}}
$$

and

$$
\frac{2\left\|x_{n+1}-x_{n}\right\|}{1+\sqrt{1+4 \frac{r_{1}-t_{n+1}}{\left(r_{1}-t_{n}\right)^{2}}\left(t_{n+1}-t_{n}\right)}} \leq\left\|x^{*}-x_{n}\right\| \leq\left(r_{1}-t_{n}\right)\left(\frac{\left\|x_{n_{0}+1}-x_{n_{0}}\right\|}{t_{n_{0}+1}-t_{n_{0}}}\right)^{2^{n-n_{0}}}
$$

are valid with

$$
t_{n+1}=t_{n}-\frac{h\left(t_{n}\right)}{h^{\prime}\left(t_{n}\right)}, t_{0}=0, \quad n=0,1, \cdots .
$$

In order to prove Theorem 3.1, we need

Proposition 3.2. Under the assumptions of Theorem 3.1, for any natural number $n \geq 1$, we have

$$
\begin{gathered}
\left\|x_{n}-x_{n-1}\right\| \leq t_{n}-t_{n-1}, \\
\left\|f^{\prime}\left(x_{0}\right)^{-1} f\left(x_{n}\right)\right\| \leq h\left(t_{n}\right)\left(\frac{\left\|x_{n}-x_{n-1}\right\|}{t_{n}-t_{n-1}}\right)^{2}, \\
\frac{\left\|f^{\prime}\left(x_{0}\right)^{-1} f\left(x_{n}\right)\right\|}{\left\|f^{\prime}\left(x_{0}\right)^{-1} f\left(x_{n-1}\right)\right\|} \leq \frac{h\left(t_{n}\right)}{h\left(t_{n-1}\right)} \cdot \frac{\left\|x_{n}-x_{n-1}\right\|}{t_{n}-t_{n-1}},
\end{gathered}
$$

and

$$
\left\|x_{n+1}-x_{n}\right\| \leq\left(t_{n+1}-t_{n}\right)\left(\frac{\left\|x_{n}-x_{n-1}\right\|}{t_{n}-t_{n-1}}\right)^{2} .
$$

Proof. By the hypotheses, (3.6) is true for $n=1$. Now assume that it holds for some $n \geq 1$. Then

$$
x_{n} \in \mathrm{B}\left(x^{*}, t_{n}\right) \subset \mathrm{B}\left(x^{*}, r_{1}\right) .
$$

Since

$$
\begin{aligned}
f\left(x_{n}\right) & =f\left(x_{n}\right)-f\left(x_{n-1}\right)-f^{\prime}\left(x_{n-1}\right)\left(x_{n}-x_{n-1}\right) \\
& =\int_{0}^{1}\left(f^{\prime}\left(x_{n-1+\tau}\right)-f^{\prime}\left(x_{n-1}\right)\right)\left(x_{n}-x_{n-1}\right) d \tau,
\end{aligned}
$$

where

we obtain

$$
x_{n-1+\tau}=x_{n-1}+\tau\left(x_{n}-x_{n-1}\right), \quad 0 \leq \tau \leq 1,
$$

$$
\left\|f^{\prime}\left(x_{0}\right)^{-1} f\left(x_{n}\right)\right\| \leq \int_{0}^{1}\left\|f^{\prime}\left(x_{0}\right)^{-1}\left(f^{\prime}\left(x_{n-1+\tau}\right)-f^{\prime}\left(x_{n-1}\right)\right)\right\| \cdot\left\|\left(x_{n}-x_{n-1}\right)\right\| d \tau .
$$


By the hypothesis (3.1), we have

$$
\begin{gathered}
\left\|f^{\prime}\left(x_{0}\right)^{-1} f\left(x_{n}\right)\right\| \leq \int_{0}^{1} \int_{\rho\left(x_{n-1}\right)}^{\rho\left(\overline{x_{n-1} x_{n-1+\tau}}\right)} L(u) d u\left\|x_{n}-x_{n-1}\right\| d \tau \\
=\int_{0}^{\left\|x_{n}-x_{n-1}\right\|} L\left(\left\|x_{n-1}-x_{0}\right\|+u\right)\left(\left\|x_{n}-x_{n-1}\right\|-u\right) d u .
\end{gathered}
$$

Since $L$ is a nondecreasing function, $\varphi(t):=\frac{1}{t^{2}} \int_{0}^{t} L(\rho+u)(t-u) d u$ is nondecreasing with respect to $t$ in $[0, r-\rho]$. In fact, when $0<t_{1}<t_{2} \leq r-\rho$, we have

$$
\begin{aligned}
\varphi\left(t_{2}\right)-\varphi\left(t_{1}\right)= & \left(\frac{1}{t_{1}^{2}}-\frac{1}{t_{2}^{2}}\right) \int_{0}^{\frac{t_{1}}{2}}\left(L\left(\rho+\frac{t_{1}}{2}+u\right)-L\left(\rho+\frac{t_{1}}{2}-u\right)\right) u d u \\
& +\frac{\left(t_{2}-t_{1}\right)^{2}}{2 t_{2}^{2} t_{1}} \int_{0}^{t_{1}}\left(L\left(\rho+t_{1}\right)-L(\rho+u)\right) d u \\
& +\frac{1}{t_{2}^{2}} \int_{t_{1}}^{t_{2}}\left(L(\rho+u)-L\left(\rho+t_{1}\right)\right)\left(t_{2}-u\right) d u \geq 0 .
\end{aligned}
$$

Hence

$$
\begin{aligned}
& \left\|f^{\prime}\left(x_{0}\right)^{-1} f\left(x_{n}\right)\right\| \\
& \leq \frac{1}{\left\|x_{n}-x_{n-1}\right\|^{2}} \int_{0}^{\left\|x_{n}-x_{n-1}\right\|} L\left(\left\|x_{n-1}-x_{0}\right\|+u\right)\left(\left\|x_{n}-x_{n-1}\right\|-u\right) d u \\
& \quad \leq \frac{1}{\left(t_{n}-t_{n-1}\right)^{2}} \int_{0}^{t_{n}-t_{n-1}} L\left(t_{n-1}+u\right)\left(t_{n}-t_{n-1}-u\right) d u\left\|x_{n}-x_{n-1}\right\|^{2} \\
& \quad=h\left(t_{n}\right)\left(\frac{\left\|x_{n}-x_{n-1}\right\|}{t_{n}-t_{n-1}}\right)^{2}
\end{aligned}
$$

where we have used the inductive hypothesis (3.6). Therefore, (3.7) holds for all $n$, which makes (3.6) hold.

Since

$$
\left\|x_{n}-x_{n-1}\right\| \leq\left\|f^{\prime}\left(x_{n-1}\right)^{-1} f^{\prime}\left(x_{0}\right)\right\| \cdot\left\|f^{\prime}\left(x_{0}\right)^{-1} f\left(x_{n-1}\right)\right\|,
$$

we obtain

$$
\frac{\left\|f^{\prime}\left(x_{0}\right)^{-1} f\left(x_{n}\right)\right\|}{\left\|f^{\prime}\left(x_{0}\right)^{-1} f\left(x_{n-1}\right)\right\|} \leq h\left(t_{n}\right) \frac{\left\|x_{n}-x_{n-1}\right\|}{\left(t_{n}-t_{n-1}\right)^{2}}\left\|f^{\prime}\left(x_{n-1}\right)^{-1} f^{\prime}\left(x_{0}\right)\right\| .
$$

By (1.2) and (1.5) we have

$$
\left\|f^{\prime}\left(x_{n-1}\right)^{-1} f^{\prime}\left(x_{0}\right)\right\| \leq \frac{1}{1-\int_{0}^{\left\|x_{n-1}-x_{0}\right\|} L(u) d u} \leq-\frac{1}{h^{\prime}\left(t_{n-1}\right)}
$$

Combining (3.12) and (3.13) and using (3.5), we get that (3.8) is also true if (3.6) is true for some $n$.

Increasing $n$ to $n+1$ in (3.11) and (3.13) and applying (3.7) and (3.13) to (3.11), we get $(3.9)$.

So (3.6) can be continued, and (3.6)-(3.9) hold for all $n \geq 1$. 
Proof of Theorem 3.1. Obviously, $\left\{t_{n}\right\}$ is convergent to $r_{1}$ monotonically. Therefore, the sequence $\left\{x_{n}\right\} \subset \mathrm{B}\left(x_{0}, r_{1}\right)$ converges. Also by $(1.1),\left\|f^{\prime}\left(x_{n}\right)\right\|$ is bounded uniformly. So from

$$
f\left(x_{n}\right)+f^{\prime}\left(x_{n}\right)\left(x_{n+1}-x_{n}\right)=0
$$

we get $\lim x_{n}=x^{*}$.

Finally, by (3.1) and

$$
\begin{aligned}
e_{n} & :=f^{\prime}\left(x_{0}\right)^{-1}\left(f\left(x^{*}\right)-f\left(x_{n}\right)-f^{\prime}\left(x_{n}\right)\left(x^{*}-x_{n}\right)\right) \\
& =\int_{0}^{1} f^{\prime}\left(x_{0}\right)^{-1}\left(f^{\prime}\left(z_{\tau}\right)-f^{\prime}\left(x_{n}\right)\right)\left(x^{*}-x_{n}\right) d \tau, \\
z_{\tau} & :=x_{n}+\tau\left(x^{*}-x_{n}\right)
\end{aligned}
$$

we obtain

$$
\begin{aligned}
\left\|e_{n}\right\| & \leq \int_{0}^{1} \int_{\rho\left(x_{n}\right)}^{\rho\left(\overline{x_{n} z_{\tau}}\right)} L(u) d u\left\|x^{*}-x_{n}\right\| d \tau \\
& =\int_{0}^{\left\|x^{*}-x_{n}\right\|} L\left(\left\|x_{n}-x_{0}\right\|+u\right)\left(\left\|x^{*}-x_{n}\right\|-u\right) d u .
\end{aligned}
$$

Since $\frac{1}{t^{2}} \int_{0}^{t} L(\rho+u)(t-u) d u$ is nondecreasing with respect to $t$, we have

$$
\begin{aligned}
\left\|e_{n}\right\| \leq & \frac{1}{\left\|x^{*}-x_{n}\right\|^{2}} \int_{0}^{\left\|x^{*}-x_{n}\right\|} L\left(\left\|x_{n}-x_{0}\right\|+u\right)\left(\left\|x^{*}-x_{n}\right\|-u\right) d u \\
\leq & \frac{1}{\left(r_{1}-t_{n}\right)^{2}} \int_{0}^{r_{1}-t_{n}} L\left(x_{n}+u\right)\left(r_{1}-t_{n} \|^{2}-u\right) d u\left\|x^{*}-x_{n}\right\|^{2} \\
& =\int_{t_{n}}^{r_{1}} L(u)\left(r_{1}-u\right) d u\left(\frac{\left\|x^{*}-x_{n}\right\|}{r_{1}-t_{n}}\right)^{2} .
\end{aligned}
$$

Therefore,

$$
\begin{aligned}
\left\|x^{*}-x_{n+1}\right\| & \leq\left\|f^{\prime}\left(x_{n}\right)^{-1} f^{\prime}\left(x_{0}\right)\right\| \cdot\left\|e_{n}\right\| \\
& \leq \frac{\int_{t_{n}}^{r_{1}} L(u)\left(r_{1}-u\right) d u}{1-\int_{0}^{t_{n}} L(u) d u}\left(\frac{\left\|x^{*}-x_{n}\right\|}{r_{1}-t_{n}}\right)^{2} .
\end{aligned}
$$

By the induction method, (3.3) follows.

By (3.9), for all $i \geq 0$ and $n \geq n_{0} \geq 0$, we have

$$
\left\|x_{n+i+1}-x_{n+i}\right\| \leq\left(t_{n+i+1}-t_{n+i}\right)\left(\frac{\left\|x_{n_{0}+1}-x_{n_{0}}\right\|}{t_{n_{0}+1}-t_{n_{0}}}\right)^{2^{n-n_{0}}} .
$$

Summing for all $i \geq 0$ results in the upper bound (3.4). It follows from (3.3) that

$$
\left\|x_{n+1}-x_{n}\right\| \leq\left\|x^{*}-x_{n}\right\|+\left\|x^{*}-x_{n+1}\right\| \leq\left\|x^{*}-x_{n}\right\|+\frac{r_{1}-t_{n+1}}{\left(r_{1}-t_{n}\right)^{2}}\left\|x^{*}-x_{n}\right\|^{2} .
$$

Then, using Gragg and Tapia [3], we obtain the proof of the lower bound (3.4). 


\section{UNDER THE PREMise OF A KANTOROVICH TYPE}

About the convergence of Newton's method, the main point of Kantorovich [2] type premise is to make

$$
h(t)=\beta-t+\frac{1}{2} L t^{2}, \quad 0 \leq t \leq R
$$

become a majorizing function. For this reason, as $\left\|x-x_{0}\right\|+\left\|x^{\prime}-x\right\| \leq r$, it is sufficient to assume that

$$
\left\|f^{\prime}\left(x_{0}\right)^{-1}\left(f^{\prime}(x)-f^{\prime}\left(x^{\prime}\right)\right)\right\| \leq L\left\|x-x^{\prime}\right\|,
$$

for a positive constant $L$. As

$$
\lambda=L \beta \leq \frac{1}{2}
$$

corresponding to (1.7), the zeros of $h$

$$
\left.\begin{array}{l}
r_{1} \\
r_{2}
\end{array}\right\}=\frac{1 \mp \sqrt{1-2 \lambda}}{L}
$$

satisfy

$$
\beta \leq r_{1} \leq 2 \beta \leq \frac{1}{L} \leq r_{2} \leq \frac{2}{L},
$$

because $r_{0}=1 / L, R=2 / L, b=1 /(2 L)$ in this case. So Theorems 1.1 and 1.5, Propositions 1.3 and 1.4 all have concrete forms. The concretization of Theorem 3.1 requires that the solution of the sequence (3.4) has a closed form

$$
t_{n}=\frac{1-q^{2^{n}-1}}{1-q^{2^{n}}} r_{1}, \quad n=0,1, \cdots,
$$

where

$$
q=\frac{1-\sqrt{1-2 \lambda}}{1+\sqrt{1-2 \lambda}} .
$$

(4.6) is independently given by [3]-[5].

For instance, the concrete forms of Theorems 1.1, 1.5 and 3.1 are, respectively,

Theorem 4.1. Let $L$ be a positive constant. Assume that $f$ satisfies the condition

$$
\left\|f^{\prime}\left(x_{0}\right)^{-1} f^{\prime}(x)-\mathrm{I}\right\| \leq L\left\|x-x_{0}\right\|, \quad \forall x \in \mathrm{B}\left(x_{0}, 1 / L\right) .
$$

Then $f_{x_{0}}^{-1}$ exists and is differentiable in the open ball

$$
\mathrm{B}\left(f\left(x_{0}\right), 1 /\left(2 L\left\|f^{\prime}\left(x_{0}\right)^{-1}\right\|\right)\right) \subset f\left(\mathrm{~B}\left(x_{0}, 1 / L\right)\right) .
$$

Moreover, the radius of this ball (the left in (4.9)) is the best possible.

Theorem 4.2. Let $L$ be a positive constant, $\beta=\left\|f^{\prime}\left(x_{0}\right)^{-1} f\left(x_{0}\right)\right\|$ and $\lambda=L \beta \leq$ $\frac{1}{2}$. Assume that $f$ satisfies the condition

$$
\left\|f^{\prime}\left(x_{0}\right)^{-1} f^{\prime}(x)-I\right\| \leq L\left\|x-x_{0}\right\|, \quad \forall x \in \overline{\mathrm{B}\left(x_{0}, r\right)},
$$

where $r_{1} \leq r<r_{2}$ if $\lambda<\frac{1}{2}$, or $r=r_{1}$ if $\lambda=\frac{1}{2}$, while $r_{1}$ and $r_{2}$ are determined by (4.4). Then the equation (0.2) has a unique solution $x^{*}$ satisfying (1.23) in the closed ball $\overline{\mathrm{B}\left(x_{0}, r\right)}$. 
Theorem 4.3. Let $L$ be a positive constant, $\beta=\left\|f^{\prime}\left(x_{0}\right)^{-1} f\left(x_{0}\right)\right\|$ and $\lambda=L \beta \leq$ $\frac{1}{2}$. Assume that $f$ satisfies the condition (4.2). Then Newton's method (0.1) is well defined for all $n$ and converges to the solution $x^{*}$ satisfying (3.2) of the equation (0.2). Moreover, for all $n \geq 0$, the best possible error bounds

$$
\left\|x^{*}-x_{n}\right\| \leq \frac{q^{2^{n}-1}}{\sum_{i=0}^{2^{n}-1} q^{i}}\left\|x^{*}-x_{0}\right\| \leq \frac{q^{2^{n}-1}}{\sum_{i=0}^{2^{n-1}-1} q^{2 i}}\left\|x_{1}-x_{0}\right\|
$$

and

$$
\begin{aligned}
\frac{2\left\|x_{n+1}-x_{n}\right\|}{1+\sqrt{1+4 q^{2^{n}} /\left(1+q^{2^{n}}\right)^{2}}} \leq\left\|x^{*}-x_{n}\right\| & \leq \frac{q}{\beta} \sum_{i=0}^{2^{n-1}-1} q^{2 i}\left\|x_{n}-x_{n-1}\right\|^{2} \\
& \leq q^{2^{n-1}}\left\|x_{n}-x_{n-1}\right\|
\end{aligned}
$$

are valid with (4.7).

Remark. It is a posterior estimation to use $\left\|x_{n}-x_{n-1}\right\|$ to estimate $\left\|x^{*}-x_{n}\right\|$. The posterior estimation in (4.12) can be obtained by setting $n_{0}=n-1$ in (3.4). In the hypothesis of Kantorovich's type, more precise posterior estimations were studied by Potra [6] and Potra \& Ptak [7].

\section{Under a PRemise of SMale type}

Under the hypotheses that $f$ is analytic and satisfies

$$
\left\|f^{\prime}\left(x_{0}\right)^{-1} f^{(n)}\left(x_{0}\right)\right\| \leq n ! \gamma^{n-1}, \quad n \geq 2,
$$

Smale [8] studied the convergence and error estimation of Newton's iteration. Wang and Han [9] (also see [10], [11]) completely improved Smale's results by introducing a majorizing function

$$
h(t)=\beta-t+\frac{\gamma t^{2}}{1-\gamma t}, \quad 0 \leq t \leq R
$$

When $\gamma\left\|x-x_{0}\right\|<1$, it is easy to derive from (5.1) that

$$
\left\|f^{\prime}\left(x_{0}\right)^{-1} f^{\prime \prime}(x)\right\| \leq h^{\prime \prime}\left(\left\|x-x_{0}\right\|\right)=\frac{2 \gamma}{\left(1-\gamma\left\|x-x_{0}\right\|\right)^{3}}
$$

(see Lemma 3 in [12] or Lemma 3.5 in [13]). Hence, conditions (1.1) and (3.1) are satisfied for the function $L$ defined by

$$
L(u)=\frac{2 \gamma}{(1-\gamma u)^{3}}
$$

Furthermore, for this $L$, the function $h$ given in (1.5) coincides with the one in (5.2).

As $\alpha=\gamma \beta \leq 3-2 \sqrt{2}$, corresponding to (1.7), the zeros of $h$

$$
\left.\begin{array}{l}
r_{1} \\
r_{2}
\end{array}\right\}=\frac{1+\alpha \mp \sqrt{(1+\alpha)^{2}-8 \alpha}}{4 \gamma}
$$

satisfy

$$
\beta \leq r_{1} \leq\left(1+\frac{1}{\sqrt{2}}\right) \beta \leq\left(1-\frac{1}{\sqrt{2}}\right) \frac{1}{\gamma} \leq r_{2} \leq \frac{1}{2 \gamma},
$$


because $r_{0}=\left(1-\frac{1}{\sqrt{2}}\right) \frac{1}{\gamma}, R=\frac{1}{2 \gamma}, b=(3-2 \sqrt{2}) \frac{1}{\gamma}$ in this case. So Theorems 1.1 and 1.5, Propositions 1.3 and 1.4 all have concrete forms. The concretization of Theorem 3.1 requires that the solution of the sequence (3.4) has a closed form

$$
t_{n}=\frac{1-q^{2^{n}-1}}{1-q^{2^{n}-1} \eta} r_{1}, \quad n=0,1, \cdots,
$$

where

$$
q=\frac{1-\alpha-\sqrt{(1+\alpha)^{2}-8 \alpha}}{1-\alpha+\sqrt{(1+\alpha)^{2}-8 \alpha}}, \quad \eta=\frac{1+\alpha-\sqrt{(1+\alpha)^{2}-8 \alpha}}{1+\alpha+\sqrt{(1+\alpha)^{2}-8 \alpha}}
$$

For instance, the concrete forms of Theorems 1.1, 1.5 and 3.1 are, respectively,

Theorem 5.1. Let $\gamma$ be a positive constant. Assume that $f$ satisfies the condition

$$
\begin{gathered}
\left\|f^{\prime}\left(x_{0}\right)^{-1} f^{\prime}(x)-I\right\| \leq \frac{1}{\left(1-\gamma\left\|x-x_{0}\right\|\right)^{2}}-1, \\
\forall x \in \mathrm{B}\left(x_{0},\left(1-\frac{1}{\sqrt{2}}\right) / \gamma\right) .
\end{gathered}
$$

Then $f_{x_{0}}^{-1}$ exists and is differentiable in the open ball

$$
\mathrm{B}\left(f\left(x_{0}\right),(3-2 \sqrt{2}) /\left(\gamma\left\|f^{\prime}\left(x_{0}\right)^{-1}\right\|\right)\right) \subset f\left(\mathrm{~B}\left(x_{0},\left(1-\frac{1}{\sqrt{2}}\right) / \gamma\right)\right) .
$$

Moreover, the radius of this ball (the left in (5.9)) is the best possible.

Theorem 5.2. Let $\gamma$ be a positive constant, $\beta=\left\|f^{\prime}\left(x_{0}\right)^{-1} f\left(x_{0}\right)\right\|$ and $\alpha=\beta \gamma \leq$ $3-2 \sqrt{2}$. Assume that $f$ satisfies the condition

$$
\left\|f^{\prime}\left(x_{0}\right)^{-1} f^{\prime}(x)-\mathrm{I}\right\| \leq \frac{1}{\left(1-\gamma\left\|x-x_{0}\right\|\right)^{2}}-1, \quad \forall x \in \overline{\mathrm{B}\left(x_{0}, r\right)},
$$

where $r_{1} \leq r<r_{2}$ if $\alpha<3-2 \sqrt{2}$, or $r=r_{1}$ if $\alpha=3-2 \sqrt{2}$, while $r_{1}$ and $r_{2}$ are determined by (5.4). Then the equation (0.2) has a unique solution $x^{*}$ satisfying (1.23) in the closed ball $\overline{\mathrm{B}\left(x_{0}, r\right)}$.

Theorem 5.3. Let $\gamma$ be a positive constant, $\beta=\left\|f^{\prime}\left(x_{0}\right)^{-1} f\left(x_{0}\right)\right\|$ and $\alpha=\beta \gamma \leq$ $3-2 \sqrt{2}$. Assume that $f$ satisfies the condition

$$
\begin{gathered}
\left\|f^{\prime}\left(x_{0}\right)^{-1}\left(f^{\prime}(x)-f^{\prime}\left(x^{\prime}\right)\right)\right\| \leq \frac{1}{\left(1-\gamma\left\|x-x_{0}\right\|-\gamma\left\|x^{\prime}-x\right\|\right)^{2}}-\frac{1}{\left(1-\gamma\left\|x-x_{0}\right\|\right)^{2}}, \\
\left\|x-x_{0}\right\|+\left\|x^{\prime}-x\right\| \leq r .
\end{gathered}
$$

Then Newton's method (0.1) is well defined for all $n$ and converges to the solution $x^{*}$ satisfying (3.2) of the equation (0.2). Moreover, for all $n \geq 0$, the best possible error bounds

$$
\begin{aligned}
\left\|x^{*}-x_{n}\right\| & \leq \frac{1-\eta}{1-q^{2^{n}-1} \eta} q^{2^{n}-1}\left\|x^{*}-x_{0}\right\| \\
& \leq \frac{1-\eta^{2}}{(1+\alpha)\left(1-q^{2^{n}-1} \eta\right)} q^{2^{n}-1}\left\|x_{1}-x_{0}\right\|
\end{aligned}
$$


and

$$
\begin{aligned}
& \frac{2\left\|x_{n+1}-x_{n}\right\|}{1+\sqrt{\left.1+4 q^{2^{n}} \frac{\left(1-q^{2^{n}}\right)\left(1-q^{2^{n}-1} \eta\right)}{\left(1-q^{2^{n+1}}-1\right.} \eta\right)^{2}}} \\
& \leq\left\|x^{*}-x_{n}\right\| \leq \frac{q\left(1-q^{2^{n}-1} \eta\right)}{r_{1}(1-\eta)}\left(\frac{1-q^{2^{n-1}-1} \eta}{1-q^{2^{n-1}}}\right)^{2}\left\|x_{n}-x_{n-1}\right\|^{2} \\
& \leq q^{2^{n-1}} \frac{1-q^{2^{n-1}-1} \eta}{1-q^{2^{n-1}}}\left\|x_{n}-x_{n-1}\right\|
\end{aligned}
$$

are valid with (5.7).

The results above can be made more general by replacing (5.3). Now we take

$$
L(u)=\frac{2 c \gamma}{(1-\gamma u)^{3}},
$$

where $c$ is a positive number. In this case the majorizing function is

$$
h(t)=\beta-t+\frac{c \gamma t^{2}}{1-\gamma t},
$$

and its zeros are

$$
\left.\begin{array}{l}
r_{1} \\
r_{2}
\end{array}\right\}=\frac{1+\alpha \mp \sqrt{(1+\alpha)^{2}-4(1+c) \alpha}}{2(1+c) \gamma}
$$

They satisfy

$$
\beta \leq r_{1} \leq\left(1+\sqrt{\frac{c}{c+1}}\right) \beta \leq\left(1-\sqrt{\frac{c}{c+1}}\right) \frac{1}{\gamma} \leq r_{2} \leq \frac{1}{(c+1) \gamma},
$$

because $r_{0}=\left(1-\sqrt{\frac{c}{c+1}}\right) \frac{1}{\gamma}, R=\frac{1}{(c+1) \gamma}, b=(1+2 c-2 \sqrt{c(c+1)}) \frac{1}{\gamma}$. Hence, we have

Theorem 5.3'. Let $\gamma$ and $c$ be positive constants, $\beta=\left\|f^{\prime}\left(x_{0}\right)^{-1} f\left(x_{0}\right)\right\|$ and $\alpha=$ $\beta \gamma \leq 1+2 c-2 \sqrt{c(c+1)}$. Assume that $f$ satisfies the condition

$$
\begin{gathered}
\left\|f^{\prime}\left(x_{0}\right)^{-1}\left(f^{\prime}(x)-f^{\prime}\left(x^{\prime}\right)\right)\right\| \leq \frac{c}{\left(1-\gamma\left\|x-x_{0}\right\|-\gamma\left\|x^{\prime}-x\right\|\right)^{2}}-\frac{c}{\left(1-\gamma\left\|x-x_{0}\right\|\right)^{2}}, \\
\left\|x-x_{0}\right\|+\left\|x^{\prime}-x\right\| \leq r .
\end{gathered}
$$

Then Newton's method (0.1) is well defined for all $n$ and converges to the solution $x^{*}$ satisfying (3.2) of the equation (0.2). Moreover, for all $n \geq 0$, the best possible error bounds (5.12) and (5.13) are valid with

$$
q=\frac{1-\alpha-\sqrt{(1+\alpha)^{2}-4(1+c) \alpha}}{1-\alpha+\sqrt{(1+\alpha)^{2}-4(1+c) \alpha}}, \quad \eta=\frac{1+\alpha-\sqrt{(1+\alpha)^{2}-4(1+c) \alpha}}{1+\alpha+\sqrt{(1+\alpha)^{2}-4(1+c) \alpha}} .
$$




\section{UNDER THE PREMISE OF ANALYTICITY}

We come back to the analytic premise about $f$, to see what stronger conclusion can be obtained. When $f$ is assumed to be analytic in the ball $\mathrm{B}\left(x_{0}, r\right), f$ can be expanded to a convergent power series

$$
f(x)=\sum_{n=0}^{\infty} \frac{1}{n !} f^{(n)}\left(x_{0}\right)\left(x-x_{0}\right)^{n} .
$$

If we suppose

$$
\left\|f^{\prime}\left(x_{0}\right)^{-1} f^{(n)}\left(x_{0}\right)\right\| \leq \gamma_{n}, \quad n \geq 2
$$

and write

$$
g(t)=\sum_{n=2}^{\infty} \frac{\gamma_{n}}{n !} t^{n}
$$

where the sequence $\gamma_{n}$ satisfies

$$
\limsup \sqrt[n]{\frac{\gamma_{n}}{n !}} \leq \frac{1}{r}
$$

then $f^{\prime}\left(x_{0}\right)^{-1} f^{\prime}$ satisfies the Lipschitz condition about $g^{\prime \prime}$ in $\mathrm{B}\left(x_{0}, r\right)$. Thus, Theorem 1.1 asserts that $f_{x_{0}}^{-1}$ exists in $\mathrm{B}\left(f\left(x_{0}\right), b /\left\|f^{\prime}\left(x_{0}\right)^{-1}\right\|\right)$ and is analytic, where

$$
b=\int_{0}^{r_{0}} g^{\prime \prime}(u) u d u=r_{0}-g\left(r_{0}\right)
$$

and $r_{0}$ satisfies

$$
\int_{0}^{r_{0}} g^{\prime \prime}(u) d u=g^{\prime}\left(r_{0}\right)=1
$$

So, we have

Theorem 6.1. Assume that $f$ is analytic in the ball $\mathrm{B}\left(x_{0}, r\right)$ and $r \geq r_{0}$. If

$$
\left\|y-f\left(x_{0}\right)\right\|<\frac{b}{\left\|f^{\prime}\left(x_{0}\right)^{-1}\right\|},
$$

then the Euler series

$$
x=x_{0}+\sum_{n=1}^{\infty} \frac{1}{n !}\left(\frac{d}{d y}\right)^{n} f_{x_{0}}^{-1}(y)_{y=f\left(x_{0}\right)}\left(y-f\left(x_{0}\right)\right)^{n}
$$

converges, and the constant $b$ in the right of (6.7), which is determined by (6.5), is the best possible.

When $\mathbf{X}=\mathbf{Y}=\mathbf{C}$, we have

Theorem 6.2. Assume that $f$ and $F$ are analytic in the open ball $\mathrm{B}\left(x_{0}, r\right) \subset \mathbf{C}$ and $r \geq r_{0}$. Then the convergence radius, $R\left(F \circ f_{x_{0}}^{-1}\right)$, of the Lagrange series

$$
\begin{aligned}
& F\left(f_{x_{0}}^{-1}(y)\right) \\
& \quad=F\left(x_{0}\right)+\sum_{n=1}^{\infty} \frac{1}{n !}\left(\frac{d}{d x}\right)^{n-1}\left(F^{\prime}(x)\left(\frac{x-x_{0}}{f(x)-f\left(x_{0}\right)}\right)^{n}\right)_{x=x_{0}}\left(y-f\left(x_{0}\right)\right)^{n}
\end{aligned}
$$


has an exact lower bound

$$
R\left(F \circ f_{x_{0}}^{-1}\right) \geq b\left|f^{\prime}\left(x_{0}\right)\right| .
$$

It is a very technical thing to choose the sequence $\left\{\gamma_{n}\right\}$ or the function $g$ such that it can give a bound of the different Taylor coefficients of $f$ and be convenient to give the values of the parameters $b$ and $r_{0}$ in Theorems 6.1 and 6.2. In this paper we propose to choose a different generating function $G$ of the unit sequence $\{1,1, \cdots\}$ with the positive constants $\gamma$ and $c$, and then the function $g$ can be obtained by

$$
g(t)=\frac{c}{G^{\prime}(0) \gamma}\left\{G(\gamma t)-G^{\prime}(0) \gamma t-G(0)\right\}
$$

Example 1 (Exponential type). Taking $G(t)=e^{t}$ as the exponential generating function of the unit sequence, we have

$$
g(t)=\frac{c}{\gamma}\left(e^{\gamma t}-\gamma t-1\right)
$$

Under the condition

$$
\left\|f^{\prime}\left(x_{0}\right)^{-1} f^{(n)}\left(x_{0}\right)\right\| \leq c \gamma^{n-1}, \quad n \geq 2
$$

we obtain that

$$
\begin{aligned}
& \gamma r_{0}=\ln \frac{c+1}{c} \\
& \gamma b=(c+1) \ln \frac{c+1}{c}-1 .
\end{aligned}
$$

Especially, as $c=1$, we have

$$
\begin{aligned}
& \gamma r_{0}=\ln 2=0.69314 \cdots, \\
& \gamma b=\ln 4-1=0.38629 \cdots
\end{aligned}
$$

Theorem 6.1 with the values above has been obtained in [14] by the method of taking the exponential generating function of the number of Schröder system as the majorizing sequence.

Example 2 (Binomial type). Taking $G(t)=1+\operatorname{sign}(m)\left\{(1-t)^{-m}-1\right\}$ as the binomial generating function of the unit sequence, where $m>-1$ and $m \neq 0$ is a real number, we have

$$
g(t)=\frac{c}{m \gamma}\left\{(1-\gamma t)^{-m}-m \gamma t-1\right\} .
$$

Under the condition

$$
\left\|f^{\prime}\left(x_{0}\right)^{-1} f^{(n)}\left(x_{0}\right)\right\| \leq c(m+1)(m+2) \cdots(m+n-1) \gamma^{n-1}, \quad n \geq 2,
$$

we obtain that

$$
\begin{aligned}
& \gamma r_{0}=1-\left(\frac{c}{c+1}\right)^{\frac{1}{m+1}}, \\
& \gamma b=1+c \frac{m+1}{m}\left(1-\left(\frac{c+1}{c}\right)^{\frac{m}{m+1}}\right) .
\end{aligned}
$$

Especially, as $m=1$ and $c=1$, we have

$$
\begin{aligned}
& \gamma r_{0}=1-\frac{1}{\sqrt{2}}=0.29289 \cdots, \\
& \gamma b=3-2 \sqrt{2}=0.17157 \cdots .
\end{aligned}
$$


Theorem 6.1 with the values above has been obtained in [13] by the method of taking the normal generating function of the number of blankets added to $n$ letters as the majorizing sequence.

Also, as $m=\frac{1}{2}, c=1$, we have

$$
\begin{aligned}
& \gamma r_{0}=1-\sqrt[3]{\frac{1}{4}}=0.37003 \cdots \\
& \gamma b=4-3 \sqrt[3]{2}=0.22023 \cdots
\end{aligned}
$$

As $m=-\frac{1}{2}, c=1$, we have

$$
\begin{aligned}
& \gamma r_{0}=\frac{3}{4} \\
& \gamma b=\frac{1}{2} .
\end{aligned}
$$

The required condition of these simple numbers is not complicated, i.e.

$$
\left\|f^{\prime}\left(x_{0}\right)^{-1} f^{(n)}\left(x_{0}\right)\right\| \leq \frac{(2 n-3) ! !}{2^{n-1}} \gamma^{n-1}, \quad n \geq 2,
$$

Example 3 (The first logarithmic type). Taking $G(t)=1-\ln (1-t)$ as the first logarithmic generating function of the unit sequence, we have

$$
g(t)=\frac{c}{\gamma} \ln \frac{1}{1-\gamma t}-c t .
$$

Under the condition

$$
\left\|f^{\prime}\left(x_{0}\right)^{-1} f^{(n)}\left(x_{0}\right)\right\| \leq c(n-1) ! \gamma^{n-1}, \quad n \geq 2,
$$

we obtain that

$$
\begin{aligned}
& \gamma r_{0}=\frac{1}{c+1}, \\
& \gamma b=1-c \ln \frac{c+1}{c} .
\end{aligned}
$$

Especially, as $c=1$, we have

$$
\begin{aligned}
& \gamma r_{0}=\frac{1}{2}, \\
& \gamma b=1-\ln 2=0.30685 \cdots .
\end{aligned}
$$

Example 4 (The second logarithmic type). Taking $G(t)=1+2 t+(1-t) \ln (1-t)$ as the second logarithmic generating function of the unit sequence, we have

$$
g(t)=\frac{c}{\gamma}(1-\gamma t) \ln (1-\gamma t)+c t
$$

Under the condition

$$
\left\|f^{\prime}\left(x_{0}\right)^{-1} f^{(n)}\left(x_{0}\right)\right\| \leq c(n-2) ! \gamma^{n-1}, \quad n \geq 2
$$

we obtain that

$$
\begin{aligned}
& \gamma r_{0}=1-e^{-\frac{1}{c}} \\
& \gamma b=1-c+c e^{-\frac{1}{c}}
\end{aligned}
$$

Especially, as $c=1$, we have

$$
\begin{aligned}
& \gamma r_{0}=1-\frac{1}{e}=0.63212 \cdots \\
& \gamma b=\frac{1}{e}=0.36787 \cdots
\end{aligned}
$$




\section{Applications to Smale's $\alpha$-Theory}

We continue the discussion of Chapter 7 in [1]. It is well known that Smale [8] first used the criterion

$$
\alpha\left(f, x_{0}\right)=\gamma\left\|f^{\prime}\left(x_{0}\right)^{-1} f\left(x_{0}\right)\right\|
$$

to judge $x_{0}$ is an approximate zero of Newton's iteration of $f$, where

$$
\gamma=\sup _{n \geq 2}\left\|\frac{1}{n !} f^{\prime}\left(x_{0}\right)^{-1} f^{(n)}\left(x_{0}\right)\right\|^{\frac{1}{n-1}} .
$$

Definition 7.1. Suppose $x_{0} \in D$ is such that Newton's iteration (0.1) is well defined for $f: D \subset X \rightarrow Y$ and satisfies

$$
e\left(x_{n}\right) \leq\left(\frac{1}{2}\right)^{2^{n-1}} e\left(x_{n-1}\right)
$$

for all positive integers $n$, where $e\left(x_{n}\right)$ denotes some measurement of the approximation degree between $x_{n}$ and $x^{*}$. Then $x_{0}$ is said to be an approximate zero of $f$ in the sense of $e\left(x_{n}\right)$.

The approximate zero defined in [8] was introduced in the sense of $\left\|x_{n+1}-x_{n}\right\|$, while the second kind of approximate zero is defined in the sense of $\left\|x^{*}-x_{n}\right\|$. Now a more reasonable definition for the second kind was introduced in [15]. We find that it is not necessary to introduce the definition of an approximate zero in the sense of $\left\|f^{\prime}\left(x_{0}\right)^{-1} f\left(x_{n}\right)\right\|$.

In fact, similarly to Theorem 7.2 in [1], by Theorem $5.3^{\prime}$ we have

Theorem 7.2. Let $\gamma, c$ and $q$ be positive numbers, $0<q<1$. Assume that $f$ satisfies the condition

$$
\begin{aligned}
& \left\|f^{\prime}\left(x_{0}\right)^{-1}\left(f^{\prime}(x)-f^{\prime}\left(x^{\prime}\right)\right)\right\| \\
& \leq \frac{c}{\left(1-\gamma\left\|x-x_{0}\right\|-\gamma\left\|x^{\prime}-x\right\|\right)^{2}}-\frac{c}{\left(1-\gamma\left\|x-x_{0}\right\|\right)^{2}} \\
& \quad \gamma\left\|x-x_{0}\right\|+\gamma\left\|x^{\prime}-x\right\| \leq 1-\sqrt{\frac{c}{c+1}}
\end{aligned}
$$

Then, as

$$
\alpha \leq \frac{2 q+c(1+q)^{2}-(1+q) \sqrt{c^{2}(1+q)^{2}+4 c q}}{2 q}
$$

for all natural numbers $n \geq 1$, it follows that

$$
\begin{gathered}
\left\|x_{n}-x^{*}\right\| \leq q^{2^{n-1}}\left\|x_{n-1}-x^{*}\right\|, \\
\left\|x_{n+1}-x_{n}\right\| \leq q^{2^{n-1}}\left\|x_{n}-x_{n-1}\right\|,
\end{gathered}
$$

and

$$
\left\|f^{\prime}\left(x_{0}\right)^{-1} f\left(x_{n}\right)\right\| \leq q^{2^{n-1}}\left\|f^{\prime}\left(x_{0}\right)^{-1} f\left(x_{n-1}\right)\right\|,
$$

where $x^{*}$ satisfies $f\left(x^{*}\right)=0$.

Especially, as

$$
\alpha\left(f, x_{0}\right) \leq \frac{4+9 c-3 \sqrt{c(9 c+8)}}{4}
$$


$x_{0}$ is an approximate zero of $f$ in any sense of $\left\|x^{*}-x_{n}\right\|,\left\|x_{n+1}-x_{n}\right\|$ or $\left\|f^{\prime}\left(x_{0}\right)^{-1} f\left(x_{n}\right)\right\|$.

Proof. The representation in the inequality (7.4) at the right side can be obtained from $\left(5.7^{\prime}\right)$ by representing $q$ by $\alpha$. Hence, under the hyposethis of (7.4), by Theorem 5.3' and Proposition 3.2, we have

$$
\begin{gathered}
\left\|x_{n}-x^{*}\right\| \leq \frac{r_{1}-t_{n}}{r_{1}-t_{n-1}}\left\|x_{n-1}-x^{*}\right\|, \\
\left\|x_{n+1}-x_{n}\right\| \leq \frac{t_{n+1}-t_{n}}{t_{n}-t_{n-1}}\left\|x_{n}-x_{n-1}\right\|
\end{gathered}
$$

and

$$
\left\|f^{\prime}\left(x_{0}\right)^{-1} f\left(x_{n}\right)\right\| \leq \frac{h\left(t_{n}\right)}{h\left(t_{n-1}\right)}\left\|f^{\prime}\left(x_{0}\right)^{-1} f\left(x_{n-1}\right)\right\| .
$$

Thus, Theorem 7.2 follows from the following lemma.

Lemma 7.3. For $\left(5.2^{\prime}\right),\left(5.4^{\prime}\right)$ and $\left(5.7^{\prime}\right)$, if $\alpha=\beta \gamma \leq 1=2 c-2 \sqrt{c(c+1)}$, then

$$
\begin{gathered}
\frac{r_{1}-t_{n}}{r_{1}-t_{n-1}}=\frac{1-q^{2^{n-1}-1} \eta}{1-q^{2^{n}-1} \eta} q^{2^{n-1}} \leq q^{2^{n-1}} \\
\frac{t_{n+1}-t_{n}}{t_{n}-t_{n-1}}=\frac{1-q^{2^{n}}}{1-q^{2^{n-1}}} \cdot \frac{1-q^{2^{n-1}-1} \eta}{1-q^{2^{n+1}-1} \eta} q^{2^{n-1}} \leq q^{2^{n-1}}
\end{gathered}
$$

and

$$
\frac{h\left(t_{n}\right)}{h\left(t_{n-1}\right)}=\frac{1-c\left(\frac{1}{\left(1-\gamma t_{n}\right)^{2}}-1\right)}{1-c\left(\frac{1}{\left(1-\gamma t_{n-1}\right)^{2}}-1\right)} \cdot \frac{t_{n+1}-t_{n}}{t_{n}-t_{n-1}} \leq q^{2^{n-1}} .
$$

Proof. As (5.2) becomes (5.2'), the representation (5.6) about $r_{1}-t_{n}$ remains true provided that $r_{1}$ and $r_{2}, q$ and $\eta$ are determined by $\left(5.4^{\prime}\right)$ and $\left(5.7^{\prime}\right)$. Hence, (7.8)(7.10) follow immediately.

Finally, similarly to Colloray 7.3 in [1], we have

Corollary 7.4. Let $\gamma$ be a posiitve number. Assume that $f^{\prime}\left(x_{0}\right)^{-1}$ exists, $f$ is analytic in $\mathrm{B}\left(x_{0}, 1 / \gamma\right)$, and for some $q \in(0,1)$

$$
\left\|\frac{1}{n !} f^{\prime}\left(x_{0}\right)^{-1} f^{(n)}\left(x_{0}\right)\right\| \leq\left(\frac{1-q}{1+q}\right)^{2} \gamma^{n-1}, \quad n \geq 2 .
$$

Then, as

$$
\alpha\left(f, x_{0}\right) \leq q
$$

(7.5) holds.

Especially, as

$$
\left\|\frac{1}{n !} f^{\prime}\left(x_{0}\right)^{-1} f^{(n)}\left(x_{0}\right)\right\| \leq \frac{\gamma^{n-1}}{9}, \quad n \geq 2
$$

and

$$
\alpha\left(f, x_{0}\right) \leq \frac{1}{2}
$$

$x_{0}$ is an approximate zero of Newton's iteration of $f$. 


\section{REFERENCES}

[1] Wang Xinghua, Convergence of Newton's method and uniqueness of the solution of equations in Banach space, Hangzhou University, preprint.

[2] L.V. Kantorovich and G.P. Akilov, Functional Analysis, Pergamon Press, 1982. MR 83h:46002

[3] W.B. Gragg and R.A. Tapia, Optimal error bounds for the Newton-Kantorovich theorem, SIAM J. Numer. Anal., 11(1974), 10-13. MR 49:8334

[4] A.M. Ostrowski, Solutions of Equations in Euclidean and Banach Spaces, Academic Press, New York, 1973. MR 50:11760

[5] Wang Xinghua, Convergence of an iterative procedure, KeXue TongBao, 20(1975), 558-559; J. of Hangzhou University, 1977, 2: 16-42; 1978, 3: 23-26.

[6] F. A. Potra, On the a posteriori error estimates for Newton's method, Beitraege Numer. Math., 12(1984), 125-138. MR 85h:65128

[7] F. A. Potra and V. Ptak, Sharp error bounds for Newton's process, Numer. Math., 34(1980), 63-72. MR 81c:65027

[8] S. Smale, Newton's method estimates from data at one point, in The Merging of Disciplines: New Directions in Pure, Applied and Computational Mathematics, R. Ewing, K.Gross and C. Martin eds, New York, Springer-Varlag, 1986, 185-196. MR 88e:65076

[9] Wang Xinghua and Han Danfu, On the dominating sequence method in the point estimates and Smale's theorem, Science in China(Ser. A.), 33(1990), 135-144. MR 91h:65081

[10] Wang Xinghua, Some results relevant to Smale's reports, in From Topology to Computation: Proceedings of the Smalefest, M.W. Hirsch, J. E. Marsden and M. Shub eds., New York, Springer-Verlag, 1993, 456-465. MR 94f:00026

[11] Wang Xinghua, A summary on complexity theorey, Contemporary Mathematics, 163(1994), 155-170. MR 94m:65007

[12] Wang Xinghua, Han Danfu and Sun Fangyu, Point estimations on deformated Newton's iteration, Math. Num. Sin., 12(1990), 145-156; Chinese J. Num. Math. Appl., 12(1990), 1-13. MR 91d:58014

[13] Wang Xinghua, Zheng Shiming and Han Danfu, Convergence on Euler's series, the iterations of Euler's and Halley's families, Acta Mathematica Sinica, 33(1990), 721-738. MR 92b:65041

[14] Wang Xinghua and Han Danfu, The convergence of Euler's series and combinatorial skills, preprint, Hangzhou University, 1996.

[15] L. Blum, F. Cucker, M. Shub and S. Smale, Complexity and Real Computation, Part II: Some Geometry of Numerical Algorithms, City University of Hong Kong, preprint, 1996.

[16] Chen Pengyuan, Approximate zeros of quadratically convergent algorithms, Mathematics of Computation, 63(1994),247-270. MR 94j:65067

Department of Mathematics, Hangzhou University, Hangzhou 310028 China 